\title{
Processing and properties of thin film pyroelectric devices
}

\author{
M. Kohli ${ }^{1}$, Y. Huang ${ }^{1}$, T. Maeder ${ }^{1}$, C. Wuethrich ${ }^{1}$, A. Bell ${ }^{1}$, P. Muralt ${ }^{1}$, N. Setter ${ }^{1}$, P. Ryser $^{2}$, \\ M. Forster ${ }^{2}$ \\ 1) Laboratoire de Céramique, EPFL, 1015 Lausanne, Switzerland \\ 2) Cerberus AG, 8708 Maennedorf, Switzerland
}

Version of record: Microelectronic Engineering 29, 93-96, 1995

http://hdl.handle.net/10.1016/0167-9317(95)00122-0

\begin{abstract}
Pyroelectric $\mathrm{PbTiO}_{3}$ thin films devices with two temperature compensating elements on a $\mathrm{SiO}_{2} / \mathrm{Si}_{3} \mathrm{~N}_{4}$ membranes have been fabricated and characterized. The measured voltage responsivity as a function of radiation modulation frequency has been compared to finite element model calculation. The relevant film properties have been compared for sputter and a sol-gel deposition techniques. The calculated responsivity amounts to $30 \mathrm{~V} / \mathrm{W}$. The measured pyroelectric signal is a few $\mu \mathrm{V}$.
\end{abstract}

\section{Introduction}

The pyroelectric effect is well established for the detection of long wavelength infra-red radiation, as simple intruder and flame detectors, or in arrays for imaging applications [1]. A number of advantages are foreseen in the use of ferroelectric thin films on semiconductor or ceramic substrates for pyroelectric detection.

The voltage per unit incident power or voltage responsivity $\left(R_{\mathrm{V}}\right)$ is the most important device characteristic $[1,2]$. For a detector in series with a unity gain, high input impedance FET, of which the capacitance is negligible, it is given by:

$$
R_{\mathrm{v}}=\left(p \cdot A \cdot \eta \cdot \omega \cdot R_{\mathrm{g}} \cdot R_{\mathrm{t}}\right) /\left[\left(1+\omega^{2} \cdot \tau_{\mathrm{e}}^{2}\right) \cdot\left(1+\omega^{2} \cdot \tau_{\mathrm{t}}^{2}\right)\right]^{0.5}
$$

in which $\mathrm{p}$ is the pyroelectric coefficient, $\mathrm{A}$ is the area and $\eta$ is the emissivity of the active element; $\omega$ is the angular modulation frequency of the input radiation. The electrical resistance of the detector is $R_{\mathrm{e}}$, and is assumed to be much greater than that of a gate bias resistor, $R_{\mathrm{g}}$, which, therefore, in combination with the electrical capacitance, $C_{\mathrm{e}}$, defines the electrical time constant of the device, $\tau_{\mathrm{e}}\left(=R_{\mathrm{g}} \cdot C_{\mathrm{e}}\right)$. Likewise, the thermal time constant, $\tau_{t}$ is defined by the thermal capacitance of the sensor, $C_{\mathrm{t}}$, and the thermal resistance to a constant temperature heat-sink, $R_{\mathrm{t}}$.

Another important device characteristic is the noise voltage $V_{\mathrm{n}}$. The noise voltage in pyroelectric detectors is mainly dominated by the Johnson noise:

$$
V_{\mathrm{n}}=\left[4 \cdot k \cdot T \cdot\left(R_{\mathrm{g}}^{-1}+\omega \cdot C_{\mathrm{e}} \cdot \tan \delta\right) \cdot \Delta f\right]^{0.5} /\left[\left(R_{\mathrm{g}}{ }^{-1}+\omega \cdot C_{\mathrm{e}} \cdot \tan \delta\right)^{2}+\omega^{2} \cdot C_{\mathrm{e}}{ }^{2}\right]^{0.5}
$$

where $\tan \delta$ is the loss tangent of the pyroelectric film, $k$ the Boltzmann constant and $\Delta f$ the frequency range.

Here we report on the properties of PT films deposited by a sol-gel technique or by sputtering, further on a IR device with a ferroelectric thin films deposed by sol-gel on a $\mathrm{SiO}_{2} / \mathrm{Si}_{3} \mathrm{~N}_{4}$ membrane. Measured characteristics are compared to that predicted by a finite element model.

\section{$2 \mathrm{PbTiO}_{3}$ thin film properties}

Table 1 shows typical observed ratio of the degree of c-axis orientation of different PT films. We see that the film orientation depends on the deposition method [3-5]. Because of the shrinking in volume during crystallisation and cooling down from $600^{\circ} \mathrm{C}$ (thermal expansion mismatch), ex-situ annealed films are tensile at the ferroelectric phase transition $\left(\sim 490^{\circ} \mathrm{C}\right)$, whereas ionic bombardment tends to impart a 
compressive stress in sputtered films. $<001>$ orientation, is favored in compressive films, due to the small $3.89 \times 3.89 \AA$ lattice "footprint". Tensile stresses conversely favor $<100>$ orientation. Films sputtered on $\mathrm{MgO}$, a high thermal expansion substrate, are under more compressive stress, and almost perfect c-axis oriented [6].

For the electrical measurements, a matrix of $0.6 \mathrm{~mm}$ diameter gold electrodes was deposited by sputtering. Measurements of capacitance and $\tan \delta$ were carried out at $1 \mathrm{kHz}$. The pyroelectric coefficient was determined by applying a temperature modulation of amplitude $1{ }^{\circ} \mathrm{C}$ and period $100 \mathrm{~s}$ to the sample whilst measuring the current with an electrometer. Films were measured both before and after poling by the application of $10 \mathrm{~V}$ for 10 minutes.

Typical results are shown in table 2. Dielectric losses of 0.018 and 0.035 have been measured for sol-gel and sputtered films respectively.

Table 1. Degree of c-axis orientation of different PT films.

\begin{tabular}{|l|c|}
\hline Sample & $<001>$ \\
\cline { 2 - 2 } & $<001>+<100>$ \\
\hline (bulk) & 0.33 \\
\hline sol-gel on Si [3] & 0.33 \\
\hline sputtered on Si [5] & 0.70 \\
\hline sputtered on Mg: [6] & 0.98 \\
\hline
\end{tabular}

Table 2. Permittivity and pyroelectric coefficient of sputtered and sol-gel PT films.

\begin{tabular}{|l|c|c|c|c|}
\hline PT films & $\varepsilon$ & \multicolumn{2}{|c|}{$p\left(\mu \mathrm{C} \cdot \mathrm{m}^{-2} \cdot \mathrm{K}^{-1}\right)$} & $p / \varepsilon$ \\
& & unpoled & poled & \\
\hline sol-gel 0.50 & 260 & 65 & 130 & 0.5 \\
\hline sputtered & 180 & 70 & 140 & 0.78 \\
\hline
\end{tabular}

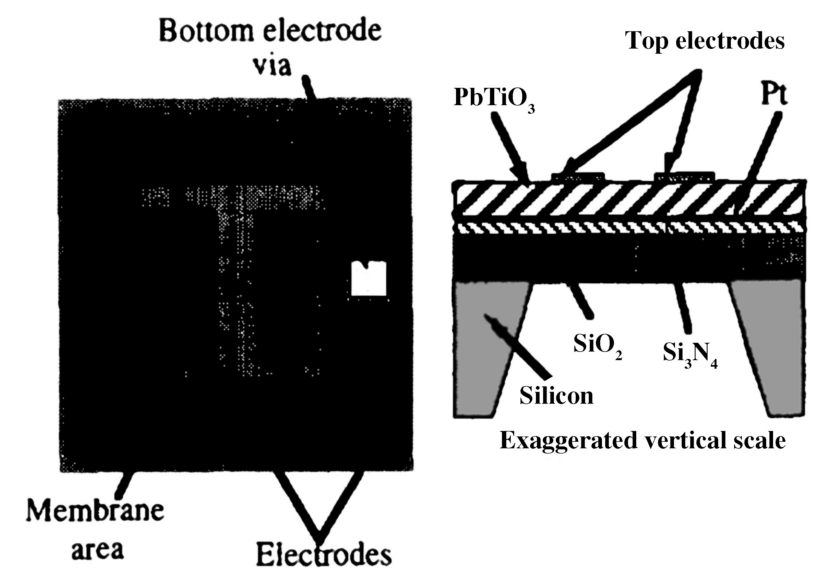

Figure 1. Dual element chip design; plan View and cross section. 


\section{Device fabrication}

Device chips were designed to fit onto a standard TO5 header; this dictates the maximum chip size to be approximately $5 \times 5 \mathrm{~mm}$. Two active areas were defined who have a common bottom electrode and are poled in the same sense with respect to the substrate.

The devices were prepared on standard, 3" diameter, $380 \mu \mathrm{m}$ thick, Si substrates as described previously [3]. The 0.5|.lm thin PT film was deposited by sol-gel on a Si substrate coated with $\mathrm{SiO}_{2} / \mathrm{Si}_{3} \mathrm{~N}_{4} /(\mathrm{Ta}) \mathrm{Pt}$. After the structuration of the $\mathrm{NiCr}$ top electrodes the $\mathrm{Si}$ was etched from the backside to get the membranes (see Figure 1). The chips were mounted and bonded with a JFET on a T05 header. Afterwards they were poled with $10 \mathrm{~V}$.

\section{Device characteristics}

Measured devices had sol-gel PT films with electrode sizes of $8.7 \mathrm{E}-7 \mathrm{~m}^{2}$ on a $2.5 \times 2.5 \mathrm{~mm}$ membrane.

\subsection{Resistance and Capacity}

Measurements were performed with a Keithley 616 digital electrometer and a HP 4274A multi frequency LCR-meter. Table 3 shows the results, where the resistance was measured with the top electrode positive $($ top + ) and negative (top -). The average of both measurements was taken for the calculation of the electrical time constant.

The resistance of the $\mathrm{PbTiO}_{3}$ thin film showed a diode and photoresistor effect. Similar effects had been observed in $\mathrm{PbLaTiO}_{3}$ thin films produced by a sol-ge1method [7].

The small PT film thickness cause a large capacitance, a third of which is parasitic nature (bonding pads).

Table 3. Capacity and resistance of one element.

\begin{tabular}{|l|c|c|c|}
\hline \multicolumn{2}{|l|}{$\mathrm{R}(\mathrm{GOhm})$} & Capacity & el. time c. \\
\hline top + & top - & C $(\mathrm{nF})$ & $\tau_{\mathrm{e}}(\mathrm{s})$ \\
\hline 303 & 39 & 4.2 & 724 \\
\hline
\end{tabular}

\subsection{Noise}

The sensor was shielded against IR-radiation, magnetic and electric noise with a $5 \mathrm{~mm}$ thick iron cap and thermally equilibrated for $15 \mathrm{~min}$. The noise of the sensor was calculated with the assumption that electronic equipment and sensor are two independent sources of white noise. The resulting noise voltage for the sensor with IFET (without equipment) amounted to $1.2 \mu \mathrm{V}$ in the frequency range of 0.05 to $25 \mathrm{~Hz}$.

\subsection{Signal}

As IR-source an emitter of about $800^{\circ} \mathrm{C}$ was focused to a spot of $0.8 \mathrm{~mm}$ which intensity was sinusoidally modulated by specific chopper and optics. The sensor was capped with an IR-transmitting filter with approximately $80 \%$ transmission in the range of 7 to $13 \mu \mathrm{m}$. For measurements a Stanford Research lock-in amplifier was used.

The measured response of the pyroelectric elements in the frequency of 1 to $12 \mathrm{~Hz}$ were at about $1.5 \mu \mathrm{V}$ (see figure 2). The reflectivity of the NiCr top electrode was measured with a Perkin-Elmer spectrometer, yielding $70 \%$. 


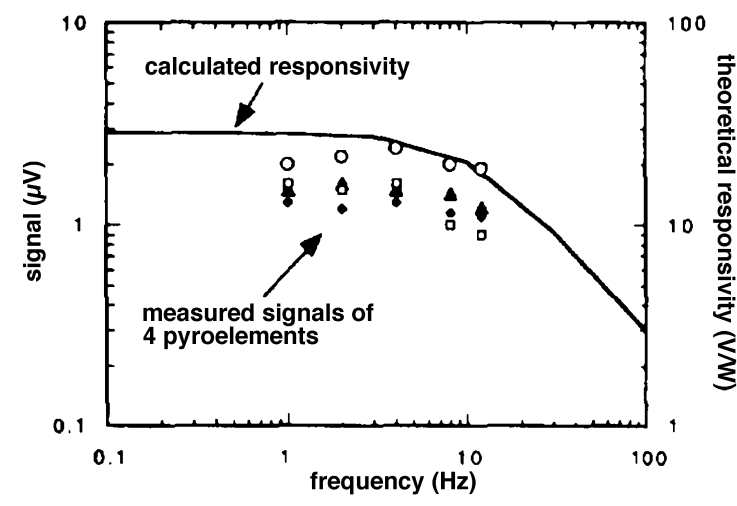

Figure 2. Signal and theoretically calculated responsivity vs. frequency.

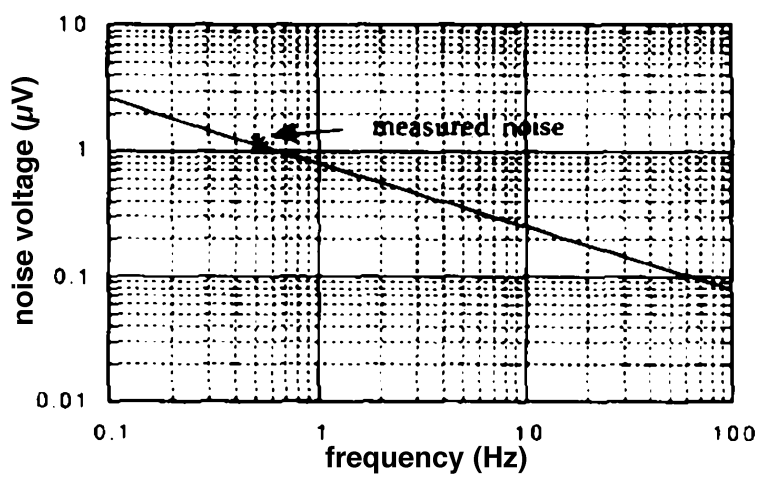

Fig. 3 Calculated and measured noise voltage vs. frequency of the device with $\Delta f=25 \mathrm{~Hz}$.

\section{Discussion}

For optimization of the sensor and comparison to obtained results, the time dependent temperature distribution was calculated by means of the finite element program ANSYS [8].

For the modeling following assumptions were made: the $\mathrm{Si}$ substrate is an infinite heat sink at room temperature, the absorption of the electrode is $100 \%$ and of the PT film $0 \%$, the membrane is in vacuum and the NiCr electrode of $25 \mathrm{~nm}$ can be neglected. The modeled sensor had two active areas of $8.7 \mathrm{E}-7 \mathrm{~m}^{2}$ on a $2.5 \times 2.5 \mathrm{~mm}$ membrane. One element was charged with a heat flux of $100 \mathrm{~W} / \mathrm{m}^{2}$.

A heat conduction in vacuum of $q_{\mathrm{cond}}=10.0 \mathrm{E}-5 \mathrm{~W} / \mathrm{K}$ was calculated from the steady-state model. The cross talk onto the second element was in the order of $25 \%$. Out of the time dependent calculation a thermal time constant of 0.025 resulted.

The heat transfer coefficient for a quiet gas environment in a small package can be approximated by:

$$
k_{\text {conv }}=k_{\mathrm{g}} /\left(1 / d_{1}+1 / d_{2}\right) \quad\left[\mathrm{W} /\left(\mathrm{m}^{2} \mathrm{~K}\right)\right]
$$

where $k_{\mathrm{g}}$ is the conduction in air and $d_{1}, d_{2}$ the distance from the membrane to the heat sink.

The losses due to radiation can be linearized:

$$
k_{\mathrm{rad}}=4 \sigma \cdot\left(\varepsilon_{1}+\varepsilon_{2}\right) \cdot T^{3} \quad\left[\mathrm{~W} /\left(\mathrm{m}^{2} \mathrm{~K}\right)\right]
$$

where $\varepsilon_{1}$ and $\varepsilon_{2}$ are the emissivities of the membrane. That yields, for the pyro element mounted in a TO5 header, to an additional heat loss of $q_{\text {conv }+ \text { rad }}=9.6 \mathrm{E}-5 \mathrm{~W} / \mathrm{K}$. The additional heat loss was included to the time dependent calculation of the heat conduction, and a thermal time constant of $0.015 \mathrm{~s}$ was evaluated.

The responsivity as function of radiation modulation frequency (see figure 2) was calculated with equation (1). With $\mathrm{p}=120 \mu \mathrm{C} \cdot \mathrm{m}^{-2} \mathrm{~K}^{-1}, R_{\mathrm{g}}=170 \mathrm{GOhm}, R_{\mathrm{t}}=\mathrm{q}_{\text {tot }}^{-1}=5102 \mathrm{~K} / \mathrm{W}, \tau_{\mathrm{e}}=724 \mathrm{~s}, \tau_{\mathrm{t}}=0.015 \mathrm{~s}$ and $\eta=30 \%$, a responsivity of $30 \mathrm{~V} / \mathrm{W}$ at $0.5 \mathrm{~Hz}$ was obtained. The measured signal showed a similar response behavior where a decrease in the responsivity after $4 \mathrm{~Hz}$ appeared. The thermal time constant is of the same order as in the model predicted.

For the two element detector with a resistance of $R_{\mathrm{g}}=340 \mathrm{GOhm}$, a capacitance of $C_{\mathrm{e}}=2.1 \mathrm{nF}$ and losses of $\tan \delta=0.02$, a noise voltage of $1.1 \mu \mathrm{V}$ at $0.05 \mathrm{~Hz}$ was calculated with equation (2). The calculated frequency dependence of the noise voltage is shown in figure 3 . This calculations were in agreement with the measurements, which indicates that the loss tangent of the sol-gel processed PT film is not much increased below at $1 \mathrm{kHz}$.

Compared with a compatible standard $\mathrm{LiTaO}_{3}$ device the signal to noise ratio has to be two orders of magnitude higher than in the present thin film device. 
Already by using a better absorber, like black Pt, a gain in the responsivity of a factor of 3 can be achieved. Further simulation showed that the responsivity will increase with a factor of 4 by using a $\mu \mathrm{m}$ thick pyroelectric thin film. The more by structuring the Pt electrode and omitting parasitic capacitance by using a isolating $\mathrm{SiO}_{2}$ layer yields in 2.5 times better responsivity. Finally by packaging the sensor into vacuum an additional factor of 3.3 will be gained. An improved sensor with a factor 100 higher responsivity is currently under development.

\section{Conclusion}

$\mathrm{PbTiO}_{3}$ films fabricated by a sol-gel method and by sputtering have been compared. Sputtered deposited films show a better c-axis orientation than films deposed by a sol-gel process. Further the figure of merit $\mathrm{p} / \varepsilon$ of sputtered PT films is 1.6 times better.

Simple film-on-membrane IR detectors, compatible with standard devices have been fabricated and characterized. The frequency response shows the same decrease, which is a result of thermal time constant as predicted from a finite element model and equation (1). Also the measured noise is equal to the one calculated with equation (2).

The responsivity can still be increased by 2 orders of magnitude by design adjustments.

\section{Acknowledgements}

This work was supported by the Swiss Priority Program, LESIT, and the Swiss Commission for Encouragement of Research, CERS.

\section{References}

[1] R.W. Whatmore, Ferroelectrics, 118 (1991) 241.

[2] M.E. Lines and A.M. Glass, Oxford Univ. Press: Oxford. (1977) 563.

[3] A. Bell, Y. Huang, M. Kohli, O. Paul, et al., Int. Symp. Appl. Ferroelectrics, (1994).

[4] Y.H. Huang, Thesis No. 1343 EPFL, Lausanne, 1995.

[5] T. Maeder and P. Muralt, Mat. Res. Soc. Symp. Proc., 341 (1994) 361.

[6] K. Iijima, Y. Tomita, R. Takayama, and I. Ueda, Jpn. J. Appl. Phys., 60(1) (1986) 361.

[7] J.J. Lee, P. Alluri, and S.K. Dey, Appl. Phys. Lett., 65(16) (1994) 2027.

[8] S.A. Sytems, SAS IP: Houston, 1994. 